\title{
BORO TOTAL E SOLÚVEL E SUAS RELAÇÕES COM ALGUNS ATRIBUTOS DOS SOLOS DO ESTADO DO CEARÁ(1)
}

\author{
F. R. SILVA ${ }^{(2)} \&$ F. F. FERREYRA H. ${ }^{(3)}$
}

\begin{abstract}
RESUMO
Foram avaliados os teores de boro total e solúvel, por meio de três extratores, usados como índices de disponibilidade para as plantas, e suas relações com as propriedades do solo em unidades de mapeamento representativas do estado do Ceará. Utilizaram-se 48 amostras compostas da camada superficial $(0-20 \mathrm{~cm})$ de solos classificados como: Areia Quartzosa (AQd), Aluvial (Ae), Planossolo (PL), Bruno Não-Cálcico (NC), Cambissolo (Ce), Podzólico Vermelho-Amarelo (PV), Latossolo Vermelho-Amarelo (LV) e Litossolo (Re). $\mathrm{O}$ boro total foi determinado após fusão com carbonato de sódio, e o boro solúvel por meio dos extratores: água quente, $\mathrm{HCl}$ 0,05 mol L-1 e Mehlich-1. O B total variou entre 8,3 e 52,2 $\mathrm{mg} \mathrm{kg}^{-1}$, com média geral de $16,0 \mathrm{mg} \mathrm{kg}^{-1}$, verificando-se as menores médias nos solos NC, Re e AQd e as maiores nos solos LV, PL e PV. Os teores de B solúvel variaram entre 0,22 e 1,22 $\mathrm{mg} \mathrm{kg}^{-1}$, com média de $0,60 \mathrm{mg} \mathrm{kg}^{-1}$, na água quente; entre 0,21 e $1,24 \mathrm{mg} \mathrm{kg}^{-1}$, com média de 0,53 $\mathrm{mg} \mathrm{kg}^{-1}$, no $\mathrm{HCl}$ 0,05 mol L-1; e entre 0,50 e 1,81 $\mathrm{mg} \mathrm{kg}^{-1}$, com média de 1,07 $\mathrm{mg} \mathrm{kg}^{-1}$, no Mehlich-1, representando 3,76; 3,32 e $6,76 \%$ do B total, respectivamente. As menores médias foram apresentadas pelos solos AQd e LV, e as maiores pelo Ce. Os três extratores correlacionaram-se de forma positiva e altamente significativa. $O$ B total e a matéria orgânica explicaram 28,8 e $38,3 \%$ do $B$ extraído pela água quente, respectivamente. $O$ conteúdo de argila, os óxidos de Mn e os sesquióxidos de Fe e Al amorfos e cristal inos influenciaram em menor proporção. A inclusão dessas características numa análise de regressão múlti pla melhorou os coeficientes de correlação.
\end{abstract}

Termos de indexação: micronutriente, boro no solo, extratores químicos, atributos do solo.

(1) Trabal ho financiado pelo CNPq. Recebido para publicação em julho de 1997 e aprovado em agosto de 1998.

(2) Pesquisadora do Departamento de Ciências do Solo do Centro de Ciências Agrárias, Universidade Federal do Ceará - UFC. Caixa Postal 12.168, CEP 60021-970 Fortaleza (CE).

(3) Professor Titular do Departamento de Ciências do Solo do Centro de Ciências Agrárias, UFC. Bolsista do CNPq. 


\title{
SUMMARY: TOTAL AND SOLUBLE BORON CONTENTS AND THEIR RELATIONSHIPS WITH SOME SOIL PROPERTIES IN THE STATE OF CEARÁ, BRAZIL
}

\begin{abstract}
Total and soluble boron contents were evailable by means of three extractors, used as plant availability indexes, in order to study their relationships with soil properties in representativemapping units of thestate of Ceará, Brazil. Compositesoil sampl es from the surface soil layers (0-20 cm) of Quartz Sands (AQd), Alluvium (Ae), Planosol (PL), Bruno non Calcic (NC), Cambisol (Ce), Red-Yel low Podzol (PV), Red-Yellow L atosol (LV) and Littosol (Re) wereused. Total boron was determi ned after fusion with sodium carbonateand sol uble boron by using the following extractors: hot water, $\mathrm{HCl} 0.05 \mathrm{~mol} \mathrm{~L}^{-1}$ and Mehlich-1. Total boron content ranged from 8.3 to $52.2 \mathrm{mg} \mathrm{kg}^{-1}$, with on average of $16.0 \mathrm{mg} \mathrm{kg}^{-1}$. The lowest averages were observed for NC, Re and AQd, and the highest for LV, PL and PV. Available boron, extracted with hot water, ranged from 0.22 to $1.22 \mathrm{mg} \mathrm{kg}^{-1}$, an average of $0.60 \mathrm{mg} \mathrm{kg}^{-1}$; for boron extracted by $\mathrm{HCl} 0.05 \mathrm{~mol} \mathrm{~L}^{-1}$, the content ranged from 0.21 to $1.24 \mathrm{mg} \mathrm{kg}^{-1}$, an average of $0.53 \mathrm{mg} \mathrm{kg}^{-1}$; for Mehlich-1, the boron content ranged from 0.50 to $1.81 \mathrm{mg} \mathrm{kg}^{-1}$, an average of $1.07 \mathrm{mg} \mathrm{kg}^{-1}$. Thesoi ls with thel owest averages wereAQd and LV and thesoil with thehighest was Ce Availableboron correlated positively in thethreechemical extractors. For hot water extraction, total B and organic matter were the main sources of available boron (28.8\% and $38.3 \%$ ) with minor influence of the clay content, $\mathrm{Mn}$ oxides, sesqui oxides of Feand Al.
\end{abstract}

Index terms: micronutrients, soil boron, chemical extractors, availability.

\section{INTRODUÇÃO}

Warington reconheceu o B como nutriente essencial para as plantas, ao encontrar que a sua deficiência restringia o crescimento radicular e ocasionava a morte das partes apicais de crescimento na cultura do feijoeiro (Russel, 1957). No Brasil, a carência de $B$ tem sido registrada em diversas culturas, tais como: o alho, (Ferrari \& ChurataMasca, 1975); a couve-flor (Ogata et al., 1979); o cafeeiro (I nstituto Brasilei ro do Café, 1974), dentre outras. Segundo Malavolta (1981), já foi constatada deficiência de B em diversas culturas de importância econômica em pelos menos dez estados brasileiros. No Ceará, informações sobre o conteúdo do boro e, em geral, dos micronutrientes no sol o são escassas, o que torna importante os levantamentos de seus teores como um meio de fornecer subsídios para a identificação de possíveis problemas de deficiência nas culturas.

O teor de B total nos solos varia amplamente e, na sua maioria, encontra-se na faixa de 4 a $98 \mathrm{mg} \mathrm{kg}^{-1}$, com média em torno de $30 \mathrm{mg} \mathrm{kg}^{-1}$ (J ackson, 1970), embora tenham sido verificados teores muito baixos, de 1 a $2 \mathrm{mg} \mathrm{kg}^{-1}$, em al guns Podzól icos da Biel orussia (Aubert \& Pinta, 1977) e valores tão altos como $1.000 \mathrm{mg} \mathrm{kg}^{-1}$, no litoral sul do Peru (Fox, 1968). No Brasil, têm sido reportados teores de $B$ total entre 10 e 34,2 mg kg-1 nos horizontes superficiais $\left(A_{1}\right)$ de sol os do município de Piracicaba (Casagrande, 1978) e de 19 a $150 \mathrm{mg} \mathrm{kg}^{-1}$, nos horizontes $\mathrm{Ap}$ ou $\mathrm{A}_{1}$, de sete grandes grupos de sol os do estado de São Paulo (Brasil Sobrinho, 1965). Segundo Malavolta (1980), na maioria dos solos brasileiros, os teores de B total encontram-se na faixa de 30 a $60 \mathrm{mg} \mathrm{kg}^{-1}$. As grandes variações do $B$ total nos sol os são determinadas, em parte, pel o material de origem e, nos diversos tipos de solos, refletem principal menteas diferenças entre as diversas regiões geográficas e zonas climáticas.

Em razão da baixa solubilidade de suas formas nativas nos solos, o $B$ total não é considerado bom índice de sua disponibilidade para as plantas. Esse fato tem levado ao desenvolvimento de diversos métodos de extração para o diagnóstico de níveis de deficiência ou suficiência de $B$ nos solos. O método de extração com água quente (Berger \& Truog, 1939), que extrai menos do $5 \%$ do B total do solo (Berger \& Truog, 1940), éum dos mais amplamente difundidos e suas determinações aceitas como índice de disponibilidade de B no solo para a maioria das plantas. Na tentativa de simplificar as análises e facilitar o seu uso em condições de rotina, têm sido empregados outros extratores como: soluções diluídas de ácidos (Ponnamperuma et al., 1981; Renan \& Gupta, 1991), soluções salinas de $\mathrm{CaCl}_{2}$ (J effery \& McCallum, 1988; Spouncer et al., 1992), soluções complexantes como manitol $+\mathrm{CaCl}_{2}$ (Cartwright et al., 1983) e AB-DTPA (Gestring \& Soltanpour, 1984, 1987). Entretanto, o método da água quente continua sendo o mais eficiente. Segundo Berger (1949), o B extraído pela água quente oscila entre 0,1 e2,0 $\mathrm{mg} \mathrm{kg}^{-1}$, enquanto, para J ackson (1970), varia de 0,2 a 1,5 $\mathrm{mg} \mathrm{kg}^{-1}$. Em solos brasileiros, os teores variam amplamente, por exemplo, de 0,06 a 0,32 $\mathrm{mg} \mathrm{kg}^{-1}$, em solos de Piracicaba, SP (Brasil Sobrinho, 1965); de 0,22 a 0,54 $\mathrm{mg} \mathrm{kg}^{-1}$, em sol os do Triângulo Mineiro, MG (Ribeiro \& Tucunango Sarabia, 1984); de 0,58 a 4,34 mg kg-1, em solos da Zona da Mata, PE (Horowitz \& Dantas, 1973). 
A disponibilidade de $B$ nos solos para as plantas é afetada, principalmente, pel os fatores: $\mathrm{pH}$, teor de matéria orgânica, tipo deargila, textura, compostos de FeeAl (Kubota et al., 1948; Parks \& White, 1952; Gupta, 1968; Sims \& Bingham, 1967, 1968; Choi \& Chen, 1979), dentreoutros. Por isso, em al guns casos, a inclusão de alguns atributos dos solos na análise de regressão pode ser importante na predição da disponibilidade de B dos solos (Gestring \& Soltanpour, 1984).

O presente trabalho teve por objetivo avaliar os teores deB total esolúvel, por meio detrês extratores químicos, em amostras compostas da camada superficial de diferentes classes de solos do estado do Ceará, assim como sua relação com alguns atributos que podem influenciar sua disponibilidade.

\section{MATERIAL E MÉTODOS}

Para avaliar o boro total e solúvel em solos do estado do Ceará, foram coletadas 48 amostras compostas da camada superficial $(0-20 \mathrm{~cm})$ de unidades representativas de solos. A seleção dos locais de amostragem foi realizada com base no Levantamento Exploratório-Reconhecimento de Sol os do estado do Ceará ( $\mathrm{l}$ acomineet al., 1973a, b). O número de amostras utilizadas por classe de solo foi: 5 de Areia Quartzosa distrófica (AQd), 5 de Aluvial eutrófico (Ae), 4 de Planossol o eutrófico (PL), 2 de Bruno Não-Cálcico (NC), 6 de Cambissolo eutrófico (Ce), 17 de Podzólico Vermelho-Amarelo distrófico (PVd) e eutrófico (PVe), 6 de Latossolo Vermelho-Amarelo eutrófico (LV) e 3 de Litossolo eutrófico (Re).
Cada amostra foi constituída de 8 a 15 subamostras retiradas ao acaso em área de, aproximadamente, $4.000 \mathrm{~m}^{2}$. A pós a coleta, as amostras foram homogeneizadas, secas em estufa a $45^{\circ} \mathrm{C}$, destorroadas e passadas em peneira de $2 \mathrm{~mm}$ de abertura. Foram realizadas análises para caracterização física e química, seguindo-se os métodos preconizados pelo SNLCS (EMBRAPA, 1997). Também foram determinados os teores dos óxidos de manganês, extraídos com hidroxilamina 0,1 mol L-1 na relação solo:extrator 1:10 (J in-Yun et al., 1987), e os teores de óxidos de ferro e alumínio amorfos, extraídos com oxalato de amônio 0,175 mol L-1 a pH 3,25 na relação solo:extrator 1:40 e agitação no escuro durante $4 \mathrm{~h}$, além das formas cristalinas por aquecimento a $100^{\circ} \mathrm{C}$ durante 30 minutos (Chao \& Zhou, 1983). Os resultados das análises das características físicas e químicas das amostras superficiais encontram-se no quadro 1.

Em todas as amostras desolo, foram determinados os teores de boro total e solúvel por meio de três extratores, usados como índices de disponibilidade para as plantas, pel os métodos descritos a seguir:

Boro total (B Total): foi determinado em extratos obtidos por fusão da amostra, em cadinho de platina, de uma mistura de $0,5 \mathrm{~g}$ de solo com 3,0 g de carbonato de sódio e dissolução do resíduo em $\mathrm{H}_{2} \mathrm{SO}_{4}$ 2,0 mol L-1, seguindo-se o procedimento indicado por J ackson (1970).

Boro solúvel em água quente $\left(\mathrm{B}-\mathrm{H}_{2} \mathrm{Oq}\right)$ : 0 boro foi extraído com água quente sob refluxo por cinco minutos, usando-se $20 \mathrm{~g}$ de solo com $40 \mathrm{~mL}$ de água desionizada. Em seguida, adicionaram-se três gotas de $\mathrm{CaCl}_{2}$ 0,1 mol L-1 e filtrou-se o material (Berger \& Truog, 1939; Bataglia \& Raij, 1990).

Quadro 1. Faixas de variação de alguns atributos, na camada superficial $(0-20 \mathrm{~cm})$, de unidades de mapeamento de solos representativas do estado do Ceará

\begin{tabular}{|c|c|c|c|c|c|c|c|c|}
\hline \multirow{2}{*}{$\begin{array}{l}\text { Atributo } \\
\text { do solo }{ }^{(1)}\end{array}$} & \multicolumn{8}{|c|}{ Classe de solo } \\
\hline & $\mathbf{A Q}$ & $\mathbf{A}$ & PL & NC & C & PV & LV & $\mathbf{R}$ \\
\hline Areia, $\mathrm{g} \mathrm{kg}^{-1}$ & $790-930$ & $210-750$ & $580-940$ & $710-840$ & $330-580$ & $300-910$ & $700-790$ & $430-810$ \\
\hline Silte, $\mathrm{g} \mathrm{kg}^{-1}$ & $40-140$ & $180-510$ & $30-250$ & $120-180$ & $170-290$ & $70-410$ & $90-170$ & $140-450$ \\
\hline Argila, $\mathrm{g} \mathrm{kg}^{-1}$ & $10-70$ & $70-280$ & $30-170$ & $40-110$ & $230-440$ & $10-350$ & $10-18$ & $50-120$ \\
\hline $\mathrm{pH} \mathrm{H}_{2} \mathrm{O}$ & $4,7-5,5$ & $5,0-7,7$ & $5,3-5,8$ & $5,7-6,2$ & $5,3-7,0$ & $4,5-7,1$ & $3,8-5,4$ & $4,9-5,7$ \\
\hline $\mathrm{MO}, \mathrm{g} \mathrm{kg}^{-1}$ & $3-10$ & $7-13$ & $4-25$ & 17 & $9-19$ & $3-35$ & $8-23$ & $4-31$ \\
\hline CTC, $\mathrm{mmol}_{\mathrm{c}} \mathrm{kg}^{-1}$ & $1,7-5,3$ & $6,9-13,4$ & $2,7-8,7$ & $4,7-7,2$ & $6,2-9,8$ & $2,0-9,9$ & $2,6-5,3$ & $2,4-11,6$ \\
\hline $\mathrm{V}, \%$ & $36-62$ & $86-100$ & $60-77$ & $58-87$ & $76-100$ & $21-96$ & $13-49$ & $42-66$ \\
\hline $\mathrm{Mn}-\mathrm{Ox}, \mathrm{mg} \mathrm{kg}^{-1}$ & $1,8-66,2$ & $99,8-286,1$ & $14,7-116,2$ & $78,7-122,8$ & $112,2-507,2$ & $1,4-342,8$ & $0,4-217,3$ & 1,3-105,3 \\
\hline Al-Ox, mg kg-1 & $261-717$ & $482-938$ & $495-560$ & $639-834$ & $1.182-1.870$ & $248-2.189$ & $860-2.085$ & $417-573$ \\
\hline $\mathrm{Fe}-\mathrm{O} \times \mathrm{A}, \mathrm{mg} \mathrm{kg}^{-1}$ & $89-290$ & $1.168-3.654$ & $412-1.957$ & $848-1.927$ & $459-965$ & $158-2.150$ & $352-902$ & $258-2.560$ \\
\hline $\mathrm{Fe}-\mathrm{O} \times \mathrm{C}, \mathrm{mg} \mathrm{kg}^{-1}$ & $193-1.970$ & $3.041-6.388$ & $763-4.312$ & $2.275-3.503$ & $1.057-4.853$ & $303-6.055$ & $1.150-4.303$ & $738-3.369$ \\
\hline
\end{tabular}

(1) MO = matéria orgânica do solo, CTC = capacidade de troca de cátions e $\mathrm{V}=$ percentagem de saturação por bases, $\mathrm{Mn}-\mathrm{Ox}=$ manganês na forma de óxido, Al-Ox = óxido de alumínio amorfo, Fe-OxA e Fe-OxC = Fe em óxidos amorfos e cristalinos, respectivamente. 
Boro solúvel em $\mathrm{HCl}$ 0,05 mol L-1 (B-HCl): foram pesados $10 \mathrm{~g}$ de solo e adicionados $20 \mathrm{~mL}$ de solução extratora, seguindo-se de cinco minutos de agitação e filtragem (Ponnamperuma et al., 1981).

Boro solúvel no extrator Mehlich-1 (BMehlich-1): foram pesados $10 \mathrm{~g}$ de solo e adicionados $20 \mathrm{~mL}$ desolução $\left(\mathrm{H}_{2} \mathrm{SO}_{4} 0,0125 \mathrm{~mol} \mathrm{~L}^{-1}+\mathrm{HCl}\right.$ $0,05 \mathrm{~mol} \mathrm{~L}-1)$, seguindo-se de quinze minutos de agitação e filtragem (Bataglia \& Raij, 1990).

Em todos os extratos, a filtração foi realizada através de papel filtroWathman 42, eo boro analisado pel o método da azometina H (Wolf, 1971, 1974).

Com base nos resultados do $B$ total, solúvel e nas características dos solos, foram realizadas análises de correl ações simples e múltiplas para determinar o grau de associação entre essas variáveis.

\section{RESULTADOS E DISCUSSÃO}

Os teores de boro total, considerando todas as amostras de solos, variaram de 8,3 a 52,2 mg kg-1, apresentando uma média geral de $16,0 \mathrm{mg} \mathrm{kg}^{-1}$ (Quadro 2). Tais resultados se encontram na faixa indicada por J ackson (1970), para a maioria dos sol os (4-98 mg kg-1). No entanto, o teor médio foi praticamente a metade do indicado pelo referido pesquisador. Dentre as amostras analisadas, 91\% apresentaram teores menores que $30 \mathrm{mg} \mathrm{kg}^{-1}$ e $83 \%$ menores que $20 \mathrm{mg} \mathrm{kg}^{-1}$ de $B$ total, valores inferiores aos da faixa indicada por Malavolta (1980) para solos brasileiros (30-60 mg kg-1). Os resultados revelam que o material de origem da maioria dos solos estudados é relativamente pobre em boro e que a variação dos teores no solo é alta ( $C V=58,6 \%)$. Os teores de B total variaram entre as classes e dentro da mesma classe de solo. As menores médias foram encontradas nos solos Brunos Não-Cálcicos, Litossolos e Areias Quartzosas, e as maiores nos Latossol os Vermelho-Amarelos, Planossolos e Podzólicos Vermelho-Amarelos. Dentre as propriedades dos sol os, a matéria orgânica foi a única que mostrou correlação simples altamente significativa com o B total (Quadro 3), explicando apenas $15 \%$ das variações do conteúdo de B total das amostras $\left(r=0,393^{* *}\right)$. A inclusão de outros atributos na análise de regressão múltipla não melhorou esse coeficiente.

Os extratores água quente e $\mathrm{HCl} 0,05 \mathrm{~mol}$ L-1 extraíram quantidades de $\mathrm{B}$ que variaram de $0,22 \mathrm{a}$ $1,22 \mathrm{mg} \mathrm{kg}^{-1}$, com média de 0,60 $\mathrm{mg} \mathrm{kg}^{-1}$, ede 0,21 a $1,24 \mathrm{mg} \mathrm{kg}^{-1}$, com média de $0,53 \mathrm{mg} \mathrm{kg}^{-1}$, respectivamente, enquanto o Mehlich-1, os teores variaram de 0,50 a 1,81 $\mathrm{mg} \mathrm{kg}^{-1}$, com média de $1,07 \mathrm{mg} \mathrm{kg}^{-1}$ (Quadro 2). Nos três extratores, os coeficientes de variação total dos teores de boro solúvel foram inferiores aos do $\mathrm{B}$ total. $\mathrm{A}$ água quente e o $\mathrm{HCl}$ apresentaram coeficientes de variação ( $C V=48,3$ e 43,3\%, respectivamente) superiores nos Mehlich-1 (CV $=29,9 \%$ ), indicando maior dispersão das amostras, característica desejável para estudos da disponíbilidade de boro. As correlações entre extratores apresentaram coeficientes lineares simples altamente significativos (Quadro 3),

Quadro 2. Amplitude de variação e teores médios de boro total e solúvel, utilizando três extratores na camada superficial $(0-20 \mathrm{~cm})$, de unidades de mapeamento de solos representativas do estado do Ceará

\begin{tabular}{|c|c|c|c|c|c|c|c|c|c|}
\hline \multirow{3}{*}{ Classe de solo } & \multirow{3}{*}{$\begin{array}{l}\text { Número de } \\
\text { amostras }\end{array}$} & & & \multicolumn{6}{|c|}{ Boro solúvel } \\
\hline & & \multicolumn{2}{|c|}{ Boro total } & \multicolumn{2}{|c|}{ Água quente } & \multicolumn{2}{|c|}{ HCl 0,05 mol L-1 } & \multicolumn{2}{|c|}{ Mehlich-1 } \\
\hline & & Amplitude & Média & Amplitude & Média & Amplitude & Média & Amplitude & Média \\
\hline & & & & & $\mathrm{mg}$ & $\mathrm{kg}^{-1}$ & & & \\
\hline AQd & 5 & $8,9-14,3$ & 12,10 & $0,25-0,45$ & 0,39 & $0,21-0,52$ & 0,33 & $0,56-0,92$ & 0,73 \\
\hline $\mathrm{Ae}$ & 5 & $9,1-18,5$ & 12,6 & $0,41-1,07$ & 0,65 & $0,38-1,24$ & 0,63 & $1,04-1,78$ & 1,24 \\
\hline NC & 2 & $8,9-12,0$ & 10,5 & $0,50-0,76$ & 0,63 & $0,38-0,56$ & 0,47 & $0,95-1,45$ & 1,20 \\
\hline $\mathrm{Ce}$ & 6 & $14,2-19,6$ & 16,7 & $0,58-0,90$ & 0,74 & $0,58-0,85$ & 0,70 & $1,26-1,51$ & 1,33 \\
\hline$P \vee d$ & 10 & $8,5-52,2$ & 18,5 & $0,22-1,22$ & 0,63 & $0,20-0,93$ & 0,52 & $0,74-1,81$ & 1,19 \\
\hline PVe & 7 & $11,0-19,9$ & 13,8 & $0,49-1,03$ & 0,65 & $0,32-0,78$ & 0,56 & $0,68-1,57$ & 0,97 \\
\hline LVd & 6 & $10,5-42,9$ & 22,3 & $0,38-0,62$ & 0,44 & $0,21-0,66$ & 0,46 & $0,50-0,96$ & 0,75 \\
\hline PLe & 4 & $8,3-44,6$ & 18,2 & $0,32-1,76$ & 0,69 & $0,26-1,04$ & 0,49 & $0,93-1,63$ & 1,13 \\
\hline $\mathrm{Re}$ & 3 & $9,4-14,1$ & 11,2 & $0,38-0,97$ & 0,58 & $0,41-0,92$ & 0,59 & $0,97-1,46$ & 1,21 \\
\hline Total & 48 & $8,3-52,2$ & 16,0 & $0,22-1,22$ & 0,60 & $0,21-1,24$ & 0,53 & $0,50-1,81$ & 1,07 \\
\hline Desvio-padrão & & & 9,38 & & 0,29 & & 0,23 & & 0,32 \\
\hline C.V. (\%) & & & 58,6 & & 48,3 & & 43,3 & & 29,9 \\
\hline
\end{tabular}


Quadro 3. Coeficientes de correlação simples (r) entre boro total e boro solúvel, utilizando três extratores e atributos, na camada superficial $(0-20 \mathrm{~cm})$, de unidades de mapeamento de solos representativas do estado do Ceará

\begin{tabular}{|c|c|c|c|c|}
\hline \multirow{2}{*}{ Variável $^{(\mathbf{1})}$} & \multicolumn{4}{|c|}{ Correlação simples $^{(2)}$} \\
\hline & B-Total & $\mathrm{B}-\mathrm{H}_{2} \mathrm{O} \mathbf{q}$ & B-HCl & B-Mehlich-1 \\
\hline B-Total, $\mathrm{mg} \mathrm{kg}^{-1}$ & - & - & - & - \\
\hline $\mathrm{B}-\mathrm{H}_{2} \mathrm{Oq}, \mathrm{mg} \mathrm{kg}^{-1}$ & $0,537 * *$ & - & - & 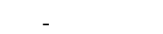 \\
\hline $\mathrm{B}-\mathrm{HCl}, \mathrm{mg} \mathrm{kg}^{-1}$ & $0,443 * *$ & $0,849 * *$ & - & - \\
\hline B-Mehlich-1, mg kg-1 & $0,286 *$ & $0,697 * *$ & $0,667 * *$ & - \\
\hline $\mathrm{pH}$ & $0,173^{\text {ns }}$ & $0,200^{\mathrm{ns}}$ & $0,317 *$ & $0,247^{\text {ns }}$ \\
\hline MO, $\mathrm{g} \mathrm{kg}^{-1}$ & $0,393 * *$ & $0,619 * *$ & $0,611 * *$ & $0,502 * *$ \\
\hline Argila, $\mathrm{g} \mathrm{kg}^{-1}$ & $0,160^{\mathrm{ns}}$ & $0,418 * *$ & $0,517 * *$ & $0,401 * *$ \\
\hline CTC, $\mathrm{mmol}_{\mathrm{Cg}^{-1}}$ & $0,051^{\text {ns }}$ & $0,440 * *$ & $0,535 * *$ & $0,600 * *$ \\
\hline Al-Ox, mg kg-1 & $0,265^{\mathrm{ns}}$ & $0,185^{\mathrm{ns}}$ & $0,364 *$ & $0,103^{\text {ns }}$ \\
\hline $\mathrm{Mn}-\mathrm{Ox}, \mathrm{mg} \mathrm{kg}^{-1}$ & $0,058^{\text {ns }}$ & $0,285 *$ & $0,337 *$ & $0,462 * *$ \\
\hline $\mathrm{Fe}-\mathrm{O} \times \mathrm{A}, \mathrm{mg} \mathrm{kg}^{-1}$ & $0,039^{\text {ns }}$ & $0,403^{* *}$ & $0,448 * *$ & $0,547 * *$ \\
\hline $\mathrm{Fe}-\mathrm{O} \times \mathrm{C}, \mathrm{mg} \mathrm{kg}^{-1}$ & $0,015^{\mathrm{ns}}$ & $0,376^{* *}$ & $0,409 * *$ & $0,427 * *$ \\
\hline \multicolumn{5}{|c|}{ 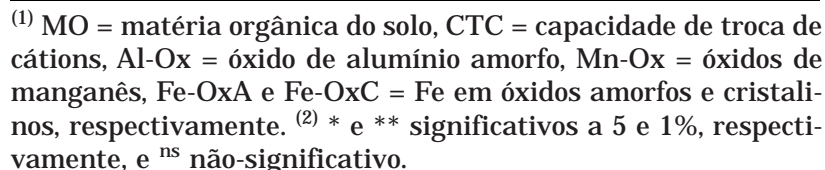 } \\
\hline
\end{tabular}

encontrando-se o valor de $r$ mais alto $(r=0,849 * *)$ entre o boro extraído pela água quente e o extraído por $\mathrm{HCl}$ 0,05 mol L-1. Com os três extratores testados, os solos que apresentaram menores teores de B solúvel foram as Areias Quartzosas e os Latossol os, sendo os maiores teores apresentados pelos Cambissolos. O B solúvel representou, em média, para todos os sol os, 3,76, 3,32 e 6,70\% do boro total, para os extratores água quente, $\mathrm{HCl}$ e Mehlich-1, respectivamente (Quadro 2). Tais resultados confirmam o trabal ho de Berger \& Truog (1940), em quea água quente extrai menos de $5 \%$ do $\mathrm{B}$ total do solo.

Os estudos de correlações simples com alguns atributos do solo indicaram que a matéria orgânica foi a que mais influiu no B solúvel do solo, seguida do borototal (Quadro 3). A matéria orgânica explicou $38,3 \%$ e o boro total $28,8 \%$ das variações nos teores de boro solúvel obtidos pel o método da água quente. Os teores de argila, óxidos de alumínio e ferro amorfos e cristalinos e os óxidos de manganês influenciaram o boro solúvel em menor proporção, enquanto o pH não teve influência sobre o $B$ solúvel dos solos estudados. Correlações positivas e significativas entre o teor de matéria orgânica e $B$ extraído pela água quente são relatadas por outros pesquisadores (Brasil Sobrinho, 1965; Gupta, 1968; Horowitz \& Dantas, 1973; Vaughan \& Howe, 1994), fato pelo qual seatribui à matéria orgânica um papel importante na disponibilidade de B, por minimizar a lixiviação do $B$ emantê-lo na forma relativamente disponível (Marzadori et al., 1991).

Quando se efetuaram correlações múltiplas entre o B solúvel e os atributos dos solos (Quadro 4), os coeficientes de correlação foram mais altos que os obtidos com as propriedades isoladas, evidenciando

Quadro 4. Equação de regressão múltipla entre boro total e boro solúvel, utilizando três extratores, e atributos na camada superficial $(0-20 \mathrm{~cm})$, de unidades de mapeamento de solos representativas do estado do Ceará

\begin{tabular}{|c|c|c|}
\hline Variável dependente & E quação de regressão(1) & Coeficiente de correlação (R) \\
\hline Boro total, mg kg-1 & $\begin{array}{l}\mathrm{Y}=20,541+1,881 \mathrm{pH}+4,216 \mathrm{MO}+0,060 \mathrm{Arg} \\
\mathrm{Y}=10,365+4,680 \mathrm{MO}-0,0012 \mathrm{Arg} \\
\mathrm{Y}=10,340+4,764 \mathrm{MO}+0,102 \mathrm{Arg}-0,0161 \mathrm{Mn}-\mathrm{Ox} \\
\mathrm{Y}=10,848+5,184 \mathrm{MO}+0,0403 \mathrm{Arg}-0,018 \mathrm{~F} \mathrm{e-OxA} \\
\mathrm{Y}=111,343+4,941 \mathrm{MO}+0,086 \mathrm{Arg}-0,001 \mathrm{Fe}-\mathrm{O} \times \mathrm{C}\end{array}$ & $\begin{array}{l}0,425^{*} \\
0,394^{*} \\
0,436^{*} \\
0,417^{*} \\
0,421^{*}\end{array}$ \\
\hline $\begin{array}{l}\text { Boro solúvel: } \\
\text { B- } \mathbf{H}_{\mathbf{2}} \mathbf{O} \mathbf{q}, \mathrm{m} \mathrm{kg}^{-1}\end{array}$ & $\begin{array}{l}Y=-0,059+0,065 \mathrm{pH}+0,210 \mathrm{MO}+0,0031 \mathrm{Arg} \\
Y=0,294+0,194 \mathrm{MO}+0,0052 \mathrm{Arg} \\
Y=0,294+0,194 \mathrm{MO}+0,0044 \mathrm{Arg}+0,00013 \mathrm{Mn}-\mathrm{Ox} \\
Y=0,282+0,181 \mathrm{MO}+0,004 \mathrm{Arg}+4,53 \mathrm{E}^{-5} \mathrm{Fe}-\mathrm{OXA} \\
\mathrm{Y}=0,276+0,189 \mathrm{MO}+0,004 \mathrm{Arg}+1,916 \mathrm{E}^{-5} \mathrm{Fe}-\mathrm{O} \mathrm{C} \mathrm{C}\end{array}$ & $\begin{array}{l}0,671 * * \\
0,645^{* *} \\
0,647 * * \\
0,656 * * \\
0,656 * *\end{array}$ \\
\hline B-HCI, mg kg-1 & $\begin{array}{l}Y=-0,152+0,077 \mathrm{pH}+0,161 \mathrm{MO}+0,0044 \mathrm{Arg} \\
Y=0,266+0,142 \mathrm{MO}+0,0069 \mathrm{Arg} \\
Y=0,266+0,141 \mathrm{MO} 0,0062 \mathrm{Arg}+0,00011 \mathrm{Mn}-\mathrm{Ox} \\
Y=0,256+0,129 \mathrm{MO}+0,0058 \mathrm{Arg}+4,320 \mathrm{E}^{-5} \mathrm{Fe}-\mathrm{O} \mathrm{AA} \\
\mathrm{Y}=0,255+0,139 \mathrm{MO}+0,0059 \mathrm{Arg}+1,166 \mathrm{E}^{-5} \mathrm{Fe}-\mathrm{O} \mathrm{C} C\end{array}$ & $\begin{array}{l}0,726 * * \\
0,677 * * \\
0,679 * * \\
0,691 * * \\
0,681 * *\end{array}$ \\
\hline B-Mehlich-1, mg kg-1 & $\begin{array}{l}\mathrm{Y}=0,307+0,087 \mathrm{pH}+0,188 \mathrm{MO}+0,004 \mathrm{Arg} \\
\mathrm{Y}=0,775+0,167 \mathrm{MO}+0,007 \mathrm{Arg} \\
\mathrm{Y}=0,774+0,162 \mathrm{MO}+0,002 \mathrm{Arg}+0,001 \mathrm{Mn}-\mathrm{Ox} \\
\mathrm{Y}=0,735+0,125 \mathrm{MO}+0,004 \mathrm{Arg}+0,0001 \mathrm{Fe}-\mathrm{OxA} \\
\mathrm{Y}=0,733+0,155 \mathrm{MO}+0,003 \mathrm{Arg}+4,326 \mathrm{E}^{-5} \mathrm{Fe}-\mathrm{O} \mathrm{CC}\end{array}$ & $\begin{array}{l}0,586 * * \\
0,546 * * \\
0,613^{* *} \\
0,637 * * \\
0,577 * *\end{array}$ \\
\hline
\end{tabular}

(1) Arg = Argila, $\mathrm{MO}=$ matéria orgânica do solo, $\mathrm{Mn}-\mathrm{Ox}$ = óxidos de manganês, Al-Ox = óxido de alumínio amorfo, Fe-OxA e Fe-OxC = Fe em óxidos amorfos e cristalinos, respectivamente. ${ }^{(2)} * \mathrm{e}^{* *}$ significativos a 5 e $1 \%$, respectivamente. 
que elas atuam de forma simultânea. Dentre os atributos analisados, o pH, a matéria orgânica e a argila, empregados simultaneamente, ou a combinação de matéria orgânica e argila nas regressões múltiplas, foram os que mais influenciaram positivamente o B extraível, principalmente nos métodos água quente e $\mathrm{HCl}$ 0,05 mol L-1. De forma similar, Chude (1986) e Gestring \& Soltanpour (1987) verificaram que a inclusão desses atributos na análise de regressão múltipla aumentou significativamente as correlações com os extratores água quente e ABDTPA. Quando adicionados, os óxidos de manganês, de ferro e de alumínio, amorfos e cristalinos, juntamente com a matéria orgânica e argila, excluindo o pH na regressão múltipla, também melhoraram os coeficientes de correlação, principalmente no extrator $\mathrm{HCl}$ 0,05 mol L-1.

Na figura 1, são apresentadas as distribuições das freqüências e a percentagem cumulativa dos solos agrupados segundo dasses de teores de $B$ removidos
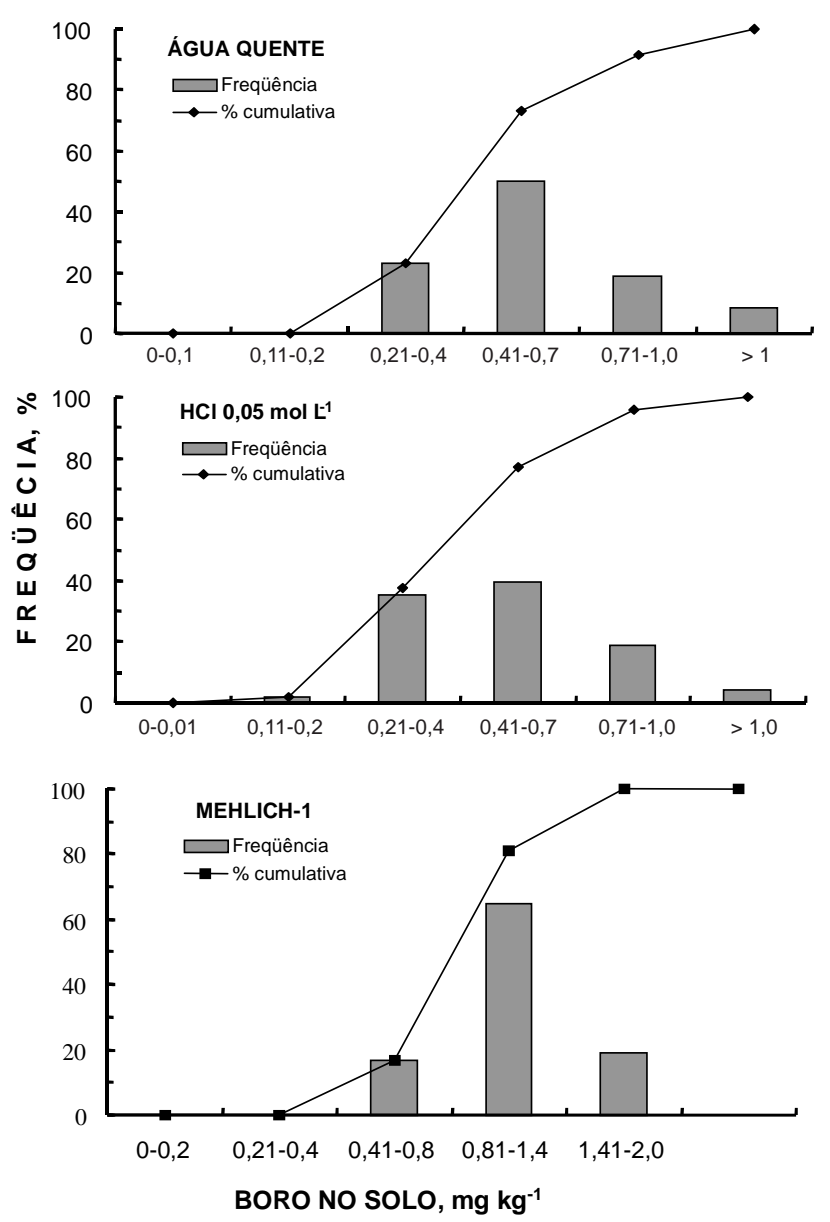

Figura 1. Freqüência e percentagem cumulativa dos teores de boro em amostras da camada superficial $(0-20 \mathrm{~cm})$ de solos do estado do Ceará, extraídos com água quente, $\mathrm{HCl}$ 0,05 mol L-1 e Mehlich-1. pelos três extratores estudados, a fim de se obter uma primeira aproximação da distribuição dos teores de B nos solos do estado do Ceará. Deve-se sal ientar que essas classes foram estabel ecidas com base em níveis críticos obtidos por outros pesquisadores em condições de solo e clima diferentes e com emprego de culturas (Siqueira et al., 1987; Bataglia \& Raij, 1990). Para a água quente e $\mathrm{HCl} 0,05 \mathrm{~mol} \mathrm{L-1}$, as classes utilizadas foram: $<0,1$ (muito baixo); 0,11 a 0,20 (baixo); 0,21 a 0,40 (médio); 0,41 a 0,70 (normal); 0,71 a 1,0 (alto) e >1,0 $\mathrm{mg} \mathrm{kg}^{-1}$ de $B$ (muito alto). Para o Mehlich-1, esses níveis foram duplicados pelo fato de esse método extrair quase o dobro de $B$ solúvel em relação aos outros dois métodos. A extração com o $\mathrm{HCl} 0,05 \mathrm{~mol} \mathrm{L-1}$ resultou em maior número de amostras com teores de $B$ inferiores a 0,4 $\mathrm{mg} \mathrm{kg}^{-1}$ comparativamente à água quente (Figura 1). I sto ocorreu porque o $\mathrm{HCl}$ $0,05 \mathrm{~mol} \mathrm{~L}^{-1}$ extraiu quantidades de boro ligeiramente menores (média de $0,53 \mathrm{mg} \mathrm{kg}^{-1}$ ). As maiores quantidades de $\mathrm{B}$ removidas pelo extrator Mehlich-1 podem ser atribuídas à mai or dissolução deóxidos de Mn, Fee deAl do sol o pela maior acidez do meio ( $50 \%$ mais $\mathrm{H}^{+}$em relaçãoao $\mathrm{HCl}$ 0,05 mol L-1), com a conseqüente liberação do $B$ nel es ocl uso. Esse fato também explica as maiores correlações verificadas entre o boro removido pel os extratores ácidos e os conteúdos dos óxidos dos solos (Quadro 3).

\section{CONCLUSÕES}

1. Os solos do estado do Ceará, em sua maior parte, apresentam conteúdos relativamente baixos em boro total, com teores na faixa de 8,3 e $52 \mathrm{mg} \mathrm{kg}^{-1}$, com média em torno a $16,0 \mathrm{mg} \mathrm{kg}^{-1}$, variando seu conteúdo amplamente entre classes e dentro da mesma classe de solos.

2. O boro solúvel é influenciado principalmente pela matéria orgânica do solo, enquanto argila, óxido de manganês e sesquióxidos de Fe e Al amorfos e cristalinos influenciam-no em menor proporção.

3. Os métodos água quente e $\mathrm{HCl} 0,05 \mathrm{~mol} \mathrm{~L}^{-1}$ extraem quantidades similares de $\mathrm{B}$ solúvel (em média 3,76 e 3,32\% do B total, respectivamente), enquanto o Mehlich-1 extrai maiores quantidades (média 6,70\% do B total). O HCl 0,05 mol L-1 distribui as amostras de sol os, pel oteor de B solúvel, em maior número de classes, o que o indica como um extrator promissor para utilização em análise de rotina.

4. Nos solos estudados, $22,4 \%$ apresentam de médios a baixos teores de $B$ solúvel e, dependendo do uso, a curto ou médio prazo, podem ser deficientes em B para a maioria das culturas. Os solos Areias Quartzosas e Latossolos são os que apresentam os menores teores de B solúvel e os Cambissolos os maiores. 


\section{LITERATURA CITADA}

AUBERT, H. \& PINTA, M. Trace elements in soils. Amsterdam, Elsevier Science, 1977. 396p.

BATAGLIA, O.C. \& RAIJ, B. van. Eficiência de extratores na determinação de boro em solos. R. Bras. Ci. Solo, 14:25-31, 1990.

BERGER, K.C. Boron in soils and crops. Adv. Agron., 1:321-351, 1949.

BERGER, K.C. \& TRUOG, E. Boron determination in soils and plants. Ind. Eng. Chem. Anal., 11:540-544, 1939.

BERGER, K.C. \& TRUOG, E. Boron deficiencies as revealed by plant and soil tests. J. Am. Sci. Agr., 32:297-301, 1940.

BRASIL SOBRINHO, M.O.C. Levantamento do teor de boro em alguns solos do estado de São Paulo. Piracicaba, Escola Superior de Agricultura Luiz de Queiroz, 1965. 135p. (Tese de Livre Docência)

CASAGRANDE,J .C. O boro em solos do município de Piracicaba. Piracicaba, Escola Superior de Agricultura “Luiz de Queiroz", 1978. 122p. (Tese de Mestrado)

CARTWRIGHT, B.; TILLER, K.G.; ZARCINAS, B.A. \& SPOUNCER, L.R. The chemical assessment of the boron status of soils. Austr. J. Soil Res., 21:321-332, 1983.

CHAO, T.T. \& ZHOU, L. Extraction techniques for selective dissolution of amorphous iron oxides from soils and sediments. Soil Sci. Soc. Am. J ., 47:225-232, 1983.

CHOI, W.W. \& CHEN, K.Y. Evaluation of boron removal by adsorption on solids. Environ. Sci. Tech., 13:189-196, 1979.

CHUDE, V.O. Relation between hot-water soluble boron and uptake of boron by cacao (Theobroma cacao L.) in Nigeria. J. Agric.Sci., 106:195-197, 1986.

EMPRESA BRASILEIRA DE PESQUISA AGROPECUÁRIA EMBRAPA. Centro Nacional de Pesquisa de Solos. Manual de métodos de análise de solos. 2.ed. Rio de J aneiro, EMBRAPA-CNPS, 1997. 212p.

FERRARI, V.A. \& CHURATA-MASCA, M.G.C. Efeitos de níveis crescentes de nitrogênio e bórax na produção de alho (Allium sativum L.). Científica, 3:254-262, 1975.

FOX, RH. The effect of calcium and $\mathrm{pH}$ on boron uptake from high concentration of boron by cotton and alfafa. Soil Sci., 106:435-439, 1968.

GESTRING, E.D. \& SOLTANPOUR, P.N. Evaluation of ammonium bicarbonate- DTPA soil test for assessing boron availability to alfafa. Soil Sci. Soc. Am. J., 48:96-100, 1984.

GESTRING, E.D. \& SOLTANPOUR, P.N. Comparison of soil tests for assessing boron toxicity to alfafa. Soil Sci. Soc. Am. J., 51:1214-1219, 1987.

GUPTA, U.C. Relationship of total and hot water soluble boron, and fixation of added boron, to properties of Podzol soils. Soil Sci. Soc. Am. Proc., 32:45-48, 1968.
HOROWITZ, A. \& DANTAS, H.S. Boro disponível nos solos da zona Litoral - Mata de Pernambuco. Pesq. Agropec. Bras., 8:163-168, 1973.

INSTITUTO BRASILEIRO DO CAFÉ - IBC. Cultura do café no Brasil. Rio de J aneiro, IBC-GE RCA, 1974. 261p.

JIN-YUN, J .; MARTENS, D.C. \& ZELAZNY, L.W. Distribution and plant availability of soil boron fractions. Soil Sci. Soc. Am. J., 51:1228-1231, 1987.

J ACKSON, M.L. Analisis químico de suelos. 2.ed. Barcelona, Omega, 1970. 662p.

JACOMINE, P.T.K.; ALMEIDA, J.C. \& MEDEIROS, L.A.R. Levantamento Exploratório - Reconhecimento de Solos do estado do Ceará. Recife, MA/DNPEA/SUDENE/DRN, 1973a. v.1. 301p. (Boletim Técnico, 28; Série Pedologia, 16)

J ACOMINE, P.T.K.; ALMEIDA, J.C. \& MEDEIROS, L.A.R. Levantamento Exploratório - Reconhecimento de Solos do Estado do Ceará. Recife, MA/DNPEA/SUDENE/DRN, 1973b. v.2. 502p. (Boletim Técnico, 28; Série Pedologia, 16)

J EFFERY,A.J \& \& MCCALLUM, L.E. Investigation of a hot 0.01M $\mathrm{CaCl}_{2}$ soil boron extraction procedure followed by ICP-AES analysis. Comm. Soil Sci. Plant Anal., 19:663-673, 1988.

KUBOTA, J .; BERGER, K.C. \& TRUOG, E. Boron movement in soils. Soil Sci. Soc. Am. Proc., 13:130-144, 1948.MALAVOLTA, E. Elementos de nutrição mineral de plantas. São Paulo, Agronômica Ceres, 1980. 251p.

MALAVOLTA, E. Manual de química agrícola; adubos eadubação. São Paulo, Agronômica Ceres, 1981. 596p.

MARZADORI, C.; ANTISARI, L.V.; CIAVATTA, C. \& SEQUI, P. Soil organic matter influence on adsorption and desorption of boron. Soil Sci. Soc. Am. J ., 55:1582-1585, 1991.

OGATA, T.; VAZ, R.L. \& FILGUEIRA, F.A.R. Efeitos de doses de bórax na produção de couve-flor de verão, em Anápolis. R. Oleric., 17:186-194, 1979.

PARKS, W.L. \& WHITE, J.L. Boron retention by clay humus systems satured with various cations. Soil Sci. Soc. Am. Proc., 16:298-300, 1952.

PONNAMPERUMA, F.N.; CAYTON, M.T. \& LANTIN, R.S. Dilute hydrochloric acid as an extractant for available zinc, copper and boron in rice soil. Plant Soil, 61:297-310, 1981.

RENAN, L. \& GUPTA, U.C. Extration of soil boron for predicting its availability to plants. Comm. Soil Sci. Plant Anal., 22:1003-1012, 1991.

RIBEIRO, A.C. \& TUCUNANGO SARABIA, W.A. Avaliação de extratores para zinco e boro disponívies em Latossolos do Triângulo Mineiro. R. Bras. Ci. Solo, 8:85-89, 1984.

RUSSEL, D.A. Boron and soil fertility, In: Sttefferud, A., ed. The yearbook of agriculture. Washington, United States Department of Agriculture, 1957. p.121-128. 
SIQUEIRA, O.J.F.; SCHERER, E.E.; TASSINARI, G.; ANGHINONI, I.;PATELLA, J .F.;TEDESCO, M.J .; MILAN, P.A. \& ERNANI, P.R. Recomendações de adubação e calagem para os estados do Rio Grande do Sul e Santa Catarina. Passo Fundo, Centro Nacional de Pesquisa do Trigo/E MBRAPA, 1987. 100p.

SIMS, J .R. \& BI NGHAM, F.T. Retention of boron by layer silicates, sesquioxides, and soil materials. I. Layer silicates. Soil Sci. Soc. Am. Proc., 31:728-732, 1967.

SIMS, J .R. \& BI NGHAM , F.T. Retention of boron by layer silicates, sesquioxides, and soil materials. II. Sesquioxides. Soil Sci. Soc. Am. Proc., 32:364-369, 1968.
SPOUNCER, L.R.; NABLE, R.O. \& CARTWRIGHT, B. A procedure for the determination of soluble boron in soils ranging widely in boron concentrations, sodicity, and $\mathrm{pH}$. Comm. Soil Sci. Plant Anal., 23:441-453, 1992.

VAUGHAN, B. \& HOWE, J. Evaluation of boron chelates in extracting soil boron. Comm. Soil Sci. Plant Anal., 25:10711084, 1994.

WOLF, B. The determination of boron in soil extracts, plant materials, composts, water and nutrient solutions. Comm. Soil Sci. Plant Anal., 2:363-374, 1971.

WOLF, B. I mprovents in the azomethine-H method for determination of boron. Comm. Soil Sci. Plant Anal., 5:3944, 1974. 